\title{
Thermohaline mixing in low-mass giants
}

\author{
M. Cantiello ${ }^{1}$ and N. Langer ${ }^{1}$ \\ ${ }^{1}$ Astronomical Institute Utrecht, Princetonplein 5, 3584 CC Utrecht, The Netherlands \\ email: M.Cantiello@uu.nl, N.Langer@uu.nl
}

\begin{abstract}
Thermohaline mixing has recently been proposed to occur in low mass red giants, with large consequences for the chemical yields of low mass stars. We investigate the role of thermohaline mixing during the evolution of stars between $1 \mathrm{M}_{\odot}$ and $3 \mathrm{M}_{\odot}$, in comparison to other mixing processes acting in these stars. We confirm that thermohaline mixing has the potential to destroy most of the ${ }^{3} \mathrm{He}$ which is produced earlier on the main sequence during the red giant stage. In our models we find that this process is working only in stars with initial mass $M \lesssim 1.5 \mathrm{M}_{\odot}$. Moreover, we report that thermohaline mixing can be present during core helium burning and beyond in stars which still have a ${ }^{3} \mathrm{He}$ reservoir. While rotational and magnetic mixing is negligible compared to the thermohaline mixing in the relevant layers, the interaction of thermohaline motions with differential rotation and magnetic fields may be essential to establish the time scale of thermohaline mixing in red giants.
\end{abstract}

Keywords. stars: evolution, stars: abundances, stars: AGB and post-AGB, stars: magnetic fields, stars: rotation, ISM: abundances

\section{Introduction}

Thermohaline mixing is not usually considered as an important mixing process in single stars, since the ashes of thermonuclear fusion consists of heavier nuclei than its fuel, and stars usually burn from the inside out. The condition for thermohaline mixing, however, is that the mean molecular weight $(\mu)$ decreases inward. Recently Charbonnel \& Zahn (2007a, CZ07) identified thermohaline mixing as an important mixing process which significantly modifies the surface composition of red giants after the first dredgeup. The work by CZ07 was triggered by the paper of Eggleton et al. (2006, EDL06), who found a $\mu$-inversion in their $1 \mathrm{M}_{\odot}$ stellar evolution model, occurring after the socalled luminosity bump on the red giant branch (RGB), which is produced after the first dredge-up, when the hydrogen-burning shell reaches the chemically homogeneous part of the envelope. The $\mu$-inversion is produced by the reaction ${ }^{3} \mathrm{He}\left({ }^{3} \mathrm{He}, 2 \mathrm{p}\right){ }^{4} \mathrm{He}$ (as predicted by Ulrich (1972)). It does not show up earlier, since the magnitude of the $\mu$-inversion is small and negligible compared to a stabilizing $\mu$-stratification.

The mixing process below the convective envelope in models of low-mass stars turns out to be essential for the prediction of the chemical yield of ${ }^{3} \mathrm{He}$ (EDL06), and to understand the surface abundances of red giants, in particular the ${ }^{12} \mathrm{C} /{ }^{13} \mathrm{C}$ ratio, and the ${ }^{7} \mathrm{Li}$, carbon and nitrogen abundances (CZ07). This may also be important for other occurrences of thermohaline mixing in stars, i.e., in single stars when a $\mu$-inversion is produced by off-center ignition in semi-degenerate cores, or in stars which accrete chemically enriched matter from a companion in a close binary (e.g., Stancliffe et al. 2007). Accreted metal-rich matter during the phases of planetary formation also leads to thermohaline mixing. The host stars of exoplanets present a metallicity excess compared to stars in which no planets have been detected. This metallicity excess can be reconciled with the overabundances expected in cases of accretion if thermohaline mixing is included in the picture (Vauclair 2004). 


\section{Method}

We use a 1-D hydrodynamic stellar evolution code (Yoon et al. 2006, and references therein). Mixing is treated as a diffusive process, the contributions to the diffusion coefficient are convection, semiconvection, thermohaline mixing, rotationally induced mixing and magnetic diffusion. The code includes the effect of centrifugal force on the stellar structure, and the transport of angular momentum is also treated as a diffusive process (Endal \& Sofia 1978; Pinsonneault et al. 1989). The condition for the occurrence of thermohaline mixing is

$$
\frac{\varphi}{\delta} \nabla_{\mu} \leqslant \nabla-\nabla_{\text {ad }} \leqslant 0
$$

i.e. the instability operates in regions that are stable against convection (according to the Ledoux criterion) and where an inversion in the mean molecular weight is present. Here $\varphi=(\partial \ln \rho / \partial \ln \mu)_{P, T}, \delta=-(\partial \ln \rho / \partial \ln T)_{P, \mu}, \nabla_{\mu}=d \ln \mu / d \ln P, \nabla_{\text {ad }}=(\partial \ln T / \partial \ln P)_{\text {ad }}$, and $\nabla=d \ln T / d \ln P$. Numerically, we treat thermohaline mixing through a diffusion scheme (Braun 1997; Wellstein et al. 2001). The corresponding diffusion coefficient is based on the work of Stern (1960), Ulrich (1972), and Kippenhahn et al. (1980):

$$
D_{t h}=-\alpha_{\mathrm{th}} \frac{3 K}{2 \rho c_{P}} \frac{\frac{\varphi}{\delta} \nabla_{\mu}}{\left(\nabla_{\mathrm{ad}}-\nabla\right)}
$$

where $\rho$ is the density, $K=4 a c T^{3} /(3 \kappa \rho)$ the thermal conductivity, and $c_{P}=(d q / d T)_{P}$ the specific heat capacity. The quantity $\alpha_{\mathrm{th}}$ is an efficiency parameter for the thermohaline mixing. The value of this parameter depends on the geometry of the fingers arising from the instability and is still a matter of debate (Ulrich 1972; Kippenhahn et al. 1980; Charbonnel \& Zahn 2007a). Most of the calculations have been performed with $\alpha_{\mathrm{th}}=2.0$, corresponding to the prescription of Kippenhahn et al. (1980), although we also investigated the effect of using different values of $\alpha_{\mathrm{th}}$.

In the code rotational mixing is included. Four different diffusion coefficients are calculated for dynamical shear, secular shear, Eddington-Sweet circulation and GoldreichSchubert-Fricke instability. Details on the physics of these instabilities and their implementation in the code can be found in Heger et al. (2000).

Chemical mixing and transport of angular momentum due to magnetic fields (Spruit 2002) is included as in Heger et al. (2000). The contribution of magnetic fields to the mixing is also calculated and added to the total diffusion coefficient $D$ entering the diffusion equation.

We compute evolutionary models of $1.0 \mathrm{M}_{\odot}, 1.5 \mathrm{M}_{\odot}, 2.0 \mathrm{M}_{\odot}$ and $3.0 \mathrm{M}_{\odot}$ at solar metallicity $(\mathrm{Z}=0.02)$. The initial equatorial velocities of these models were chosen to be 10 , 45,140 and $250 \mathrm{~km} \mathrm{~s}^{-1}$ (Tassoul 2000); we assume the stars are rigidly rotating at the zero-age main sequence. Throughout the evolution of all models, the mass-loss rate of Reimers (1975) was used.

\section{Results}

We confirm the presence of an inversion in the mean molecular weight in the outer wing of the H-burning shell, after the luminosity bump on the red giant branch. According to inequality (2.1) this inversion gives rise to thermohaline mixing in the radiative buffer layer, the radiative region between the H-burning shell and the convective envelope.

Results of our calculations for the 1.0 and $2.0 \mathrm{M}_{\odot}$ models are shown in Fig. 1 . In our $1 \mathrm{M}_{\odot}$ model, thermohaline mixing develops at the luminosity bump and transports chemical species in the radiative layer between the H-burning shell and the convective envelope. 

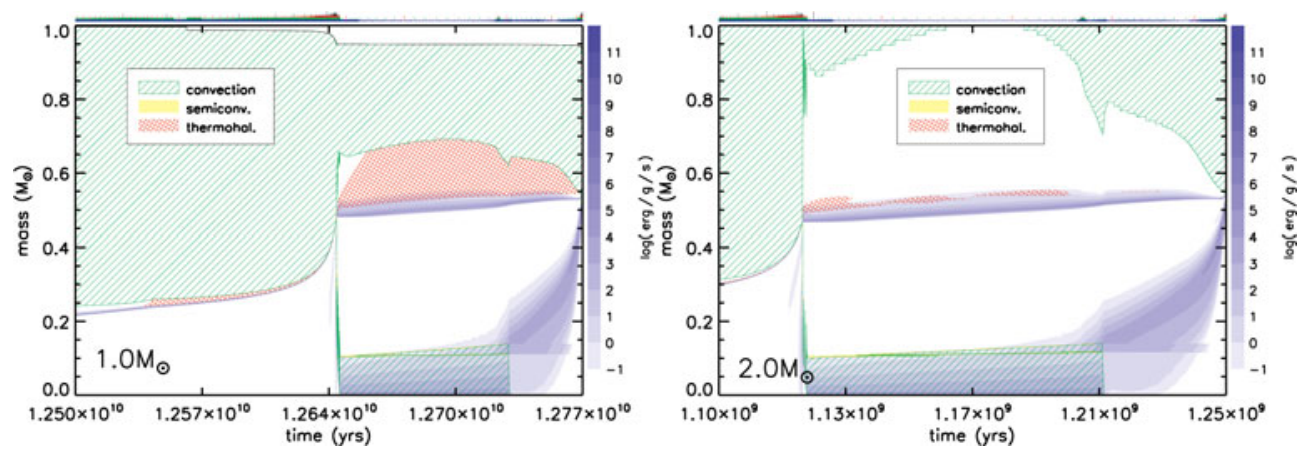

Figure 1. Left: evolution of the internal structure of a $1.0 \mathrm{M}_{\odot}$ star from the onset of thermohaline mixing to the AGB phase. Green hatched regions indicate convection, yellow filled regions represent semiconvection and red cross hatched regions indicate thermohaline mixing, as displayed in the legend. Blue shading shows regions of nuclear energy generation. Right: same as left panel, but for a $2.0 \mathrm{M}_{\odot}$ star.

This results in a change to the stellar surface abundances. The left panel of Fig. 2 shows the evolution of the ${ }^{3} \mathrm{He}$ surface abundance, and of the ratio ${ }^{12} \mathrm{C} /{ }^{13} \mathrm{C}$ at the surface as a function of time in the $1 \mathrm{M}_{\odot}$ model, confirming the result of EDL06 and CZ07, namely that thermohaline mixing is efficiently depleting ${ }^{3} \mathrm{He}$ and lowering the ratio ${ }^{12} \mathrm{C} /{ }^{13} \mathrm{C}$ on the giant branch. Interestingly the $2 \mathrm{M}_{\odot}$ model shows a different behaviour: thermohaline mixing is not able to connect the $\mathrm{H}$-burning shell and the convective envelope, resulting in no change to the surface abundance of ${ }^{3} \mathrm{He}$ and to the ratio ${ }^{12} \mathrm{C} /{ }^{13} \mathrm{C}$ after the luminosity bump. Our models with $\alpha_{\mathrm{th}}=2.0$ show thermohaline mixing to be important during the RGB only in stars with mass $M \lesssim 1.5 \mathrm{M}_{\odot}$.

While CZ07 and EDL07 investigate thermohaline mixing only during the RGB phase, we followed the evolution of our models until the thermally pulsating AGB phase (TPAGB). In fact a $\mu$-inversion is always created if a $\mathrm{H}$-burning shell is active in a chemically homogeneous layer; this happens not only during the RGB phase, but also during the horizontal branch (HB) and the AGB. The size of the $\mu$-inversion depends on the local amount of ${ }^{3} \mathrm{He}$. As a consequence, the efficiency of thermohaline mixing in different evolutionary phases depends on the amount of ${ }^{3} \mathrm{He}$ left by previous mixing episodes.

After the core He-flash, helium is burned in the core, while a H-burning shell is active below the convective envelope. We found that during this phase thermohaline mixing is present and can spread through the whole radiative buffer layer in our $1 \mathrm{M}_{\odot}$ model (left panel in Fig. 1). In this model the surface abundances change also during this phase because the H-burning shell and the envelope are connected. This is shown in Fig. 2, left panel, where surface abundances change after the luminosity peak corresponding to the He-flash. We stress that using the prescription of Kippenhahn et al. (1980) for thermohaline mixing allows our model to reach this phase without completely burning the ${ }^{3} \mathrm{He}$; models of CZ07 almost completely deplete ${ }^{3} \mathrm{He}$ in the envelope during the RGB phase because of their higher diffusion coefficient (Ulrich 1972). In this case thermohaline mixing would be much less efficient, during the subsequent evolutionary phases, due to the lower abundance of ${ }^{3} \mathrm{He}$.

The last nuclear-burning phase of a low-mass star is the AGB, and is characterized by the presence of two burning shells and a degenerate core. The star burns $\mathrm{H}$ in a shell and the ashes of this process feed an underlying He shell. During the most luminous part of the AGB the He shell periodically experiences thermal pulses (TPs); in stars more massive than about $1.2 \mathrm{M}_{\odot}$ these TPs are associated with a deep penetration of the 

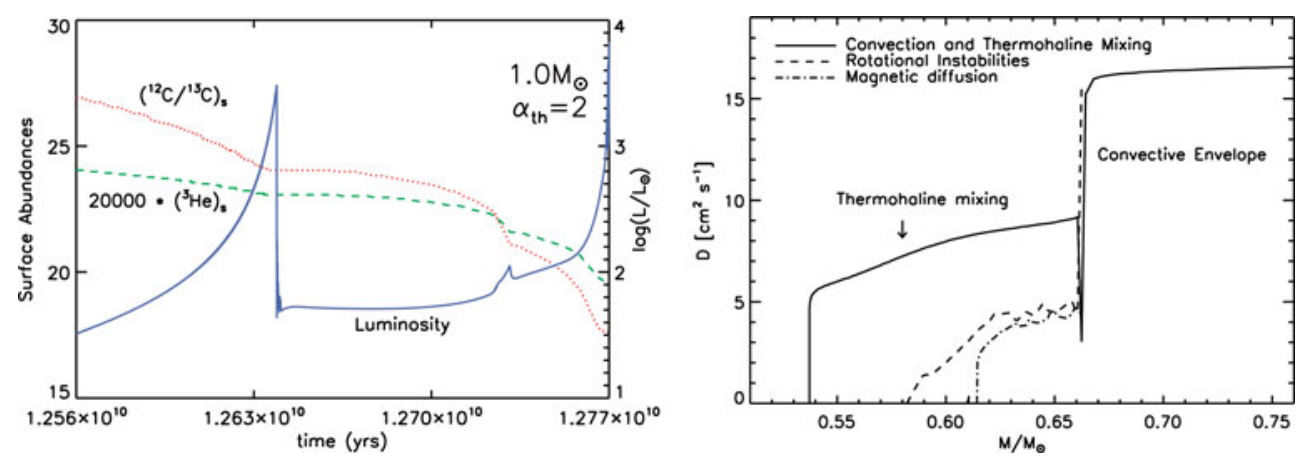

Figure 2. Left: evolution of the surface abundance of the ${ }^{12} \mathrm{C} /{ }^{13} \mathrm{C}$ ratio (dotted red line) and ${ }^{3} \mathrm{He}$ (dashed green line), and of the luminosity (solid blue line) from the onset of thermohaline mixing up to the AGB phase for a $1.0 \mathrm{M}_{\odot}$ star. Right: diffusion coefficients in the region between the $\mathrm{H}$ burning shell and the convective envelope for the $1.0 \mathrm{M}_{\odot}$ model during core He-burning $\left(\mathrm{t}=1.267 \times 10^{10}\right.$ years $)$. The black, continuous line shows convective and thermohaline mixing diffusion coefficients, the green, dashed line is the sum of the diffusion coefficients due to rotational instabilities while the blue, dot-dashed line shows the magnitude of magnetic diffusion coefficient.

convective envelope, the so-called third dredge-up (3DUP) (Wallerstein \& Knapp 1998). We find thermohaline mixing to be present during the TP-AGB phase. Depending on the mass of the model the diffusion process is able to connect the H-burning shell with the convective envelope during the whole interpulse phase. In a $1 \mathrm{M}_{\odot}$ model thermohaline mixing connects the H-burning shell to the convective envelope (Fig. 3, left panel), confirming that this mixing process is more efficient at lower masses. The occurrence of thermohaline mixing in this late evolutionary phase is critically determined by the mixing history of the star. This is because the amount of ${ }^{3} \mathrm{He}$ present during the TP-AGB phase is controlled by the occurrence of thermohaline mixing and by its efficiency $\left(\alpha_{\text {th }}\right)$ in previous evolutionary phases.

Thermohaline mixing during the TP-AGB is a circulation of the type inferred by Cameron \& Fowler (1971), since it carries ${ }^{7}$ Be out of the high-temperature zone. ${ }^{7}$ Be can then produce ${ }^{7} \mathrm{Li}$ by electron capture, which is transported in the convective envelope. This results in surface enrichment of ${ }^{7} \mathrm{Li}$, as shown in the right panel of Fig. 3. Interestingly Uttenthaler et al. (2007) recently reported the detection of low-mass, Li-rich AGB stars in the galactic bulge. Given their low mass, these stars are not expected to experience any hot bottom burning. We argue that thermohaline mixing is a possible explanation for their abundance anomalies.

\section{Rotation and magnetic fields}

In our models we found that in the relevant layers thermohaline mixing has generally higher diffusion coefficients than rotational instabilities and magnetic diffusion. This result is not valid for magnetic stars, stars that possess anomalous surface fields of a few $10^{2}$ to about $10^{4} \mathrm{G}$, believed to be of fossil origin (Charbonnel \& Zahn 2007b). The right panel of Fig. 2 clearly shows that rotational and magnetic mixing are negligible compared to the thermohaline mixing in our $1.0 \mathrm{M}_{\odot}$ model. The only rotational instability acting on a shorter timescale is the dynamical shear instability, visible in the right panel of Fig. 2 (dashed line) as a spike present at the lower boundary of the convective envelope. This instability works on the dynamical timescale in regions of a star characterized by a high degree of differential rotation. However, if present, this instability acts only in a very 

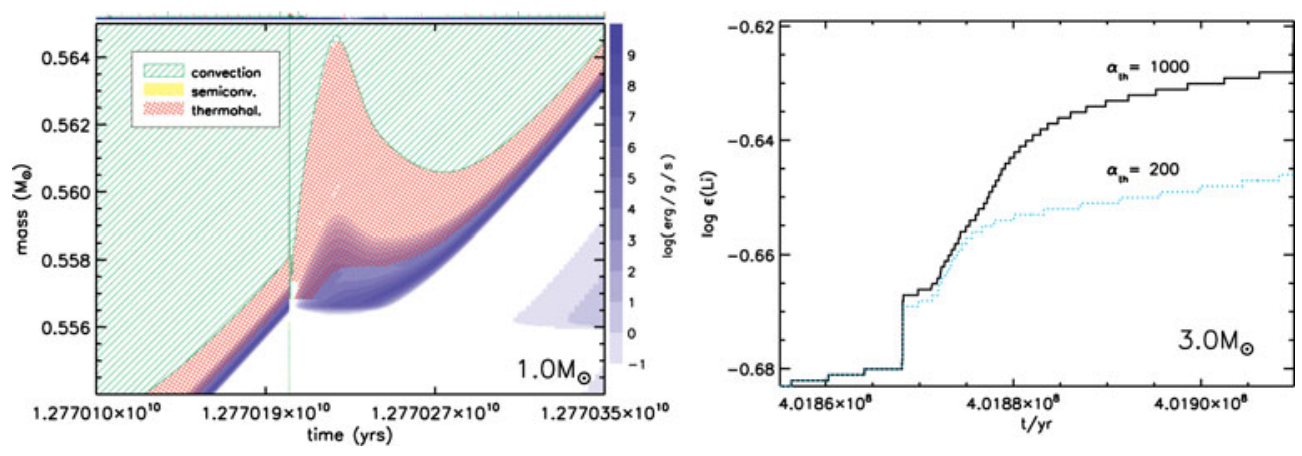

Figure 3. Left: evolution of the region between the $\mathrm{H}$ burning shell source and the convective envelope during a thermal pulse in a $1.0 \mathrm{M}_{\odot}$ star. Green hatched regions indicate convection and red crossed regions indicate thermohaline mixing. Blue shading shows regions of nuclear energy generation. Right: evolution of Lithium surface abundance during one thermal pulse in a $3 \mathrm{M}_{\odot}$ model. The black, continuous line shows a model evolved with $\alpha_{\mathrm{th}}=1000$; the blue, dotted line refers to the same model evolved with a thermohaline mixing efficiency $\alpha_{\text {th }}=200$. In both cases the model is experiencing third dredge-up. The evolution of the star prior to the TP-AGB has been calculated with $\alpha_{\mathrm{th}}=2$.

small region (in mass coordinate) at the bottom of the convective envelope. As a result thermohaline mixing is still setting the timescale for the diffusion of chemical species from the convective envelope to the hydrogen-burning shell.

The interaction between rotation and thermohaline mixing is more difficult to address, since it requires full hydrodynamic calculations. We expect the speed of thermohaline mixing to be affected by differential rotation and rotational instabilities, since these are able to change the geometry of the fingers, and to create turbulence, respectively. Canuto et al. (2008) recently suggested that turbulence must be taken into account to explain both laboratory and ocean data of double diffusive processes.

The interaction of magnetic fields with thermohaline mixing must also be considered. This depends on the geometry and the magnitude of the magnetic field: if the magnetic field is stronger or of the same order of the equipartition value the magnetic field allows plasma motions only along the direction of the field lines. This could result in the inhibition of thermohaline mixing, as discussed with detailed calculations by Zahn and Charbonnel (2008). However strong magnetic fields are observed in only a small fraction of low-mass stars (Wolff (1968); Power et al. (2007)). Concerning the interaction of weak fields with thermohaline motions, a change in the speed of the fingers is also expected and fully MHD calculations are needed to understand the process.

\section{Discussion}

We comfirm the results of EDL06 and CL07: thermohaline mixing in low-mass giants is capable of destroying large quantities of ${ }^{3} \mathrm{He}$, as well as decreasing the ratio ${ }^{12} \mathrm{C} /{ }^{13} \mathrm{C}$. Thermohaline mixing starts when the hydrogen burning shell moves into the chemically homogeneous layers established by the first dredge-up. Our models show further that thermohaline mixing remains important during core helium burning, and can still be relevant during the AGB phase - including the termally-pulsing AGB stage. This can result in important changes to the surface abundances of low-mass stars. The quantitative discussion is complicated by the fact that the capability of thermohaline mixing to change surface abundances depends on an uncertain efficiency parameter, as well as on the local ${ }^{3} \mathrm{He}$ abundance. The efficiency parameter $\alpha_{\mathrm{th}}$ is still a matter of debate and is probably 
strongly affected by the interaction of thermohaline mixing with rotation and magnetic fields. The local ${ }^{3} \mathrm{He}$ abundance depends on the previous history of mixing.

Our calculations show that in the relevant layers, thermohaline mixing generally has a higher diffusion coefficient than rotational instabilities and magnetic diffusion. Still, the interaction of thermohaline mixing with magneto-rotational instabilities is important: we expect the speed of the mixing to be strongly affected by the presence of differential rotation and magnetic fields. To better understand the picture it would be desirable to have realistic MHD simulations of thermohaline mixing.

\section{Acknowledgements}

MC wishes to thank V. Canuto, J.-P. Zahn and C. Charbonnel for useful discussions. $\mathrm{MC}$ acknowledges LKBF and the IAU for financial support.

\section{References}

Braun, H. 1997, PhD thesis, Ludwig-Maximilians-Univ. München

Cameron, A. G. W. \& Fowler, W. A. 1971, ApJ, 164, 111

Canuto, V. M., Cheng, Y., \& Howard, A. M. 2008, Geophysical Research Letters, 35, 2

Charbonnel, C. \& Zahn, J.-P. 2007, A\& A, 467, L15

Charbonnel, C. \& Zahn, J.-P. 2007, A\& A, 476, L29

Eggleton, P. P., Dearborn, D. S. P., \& Lattanzio, J. C. 2006, Science, 314, 1580

Endal, A. S. \& Sofia, S. 1978, ApJ, 220, 279

Heger, A., Langer, N., \& Woosley, S. E. 2000, ApJ, 528, 368

Kippenhahn, R., Ruschenplatt, G., \& Thomas, H.-C. 1980, A\&A, 91, 175

Pinsonneault, M. H., Kawaler, S. D., Sofia, S., \& Demarque, P. 1989, ApJ, 338, 424

Power, J., Wade, G. A., Hanes, D. A et al. 2007, Physics of Magnetic Stars, 89

Reimers, D. 1975, Memoires of the Societe Royale des Sciences de Liege, 8, 369

Spruit, H. C. 2002, A\&\&A, 381, 923

Stancliffe, R. J., Glebbeek, E., Izzard, R. G., \& Pols, O. R. 2007, A\&A, 464, L57

Tassoul, J.-L. 2000, Stellar Rotation, Cambridge University Press, 36

Ulrich, R. K. 1972, ApJ, 172, 165

Uttenthaler, S., Lebzelter, T., Palmerini, S. et al. 2007, A\&A, 471, L41

Vauclair, S. 2004, ApJ, 605, 874

Wallerstein, G. \& Knapp, G.R 1998, ARAA, 36, 369

Wellstein, S., Langer, N., \& Braun, H. 2001, A\&A, 369, 939

Wolff, S. C. 1968, PASP, 80, 281

Yoon, S.-C., Langer, N., \& Norman, C. 2006, A\&A, 460, 199

R. WALDMAN: Another possible case where thermohaline mixing can occur is off-center carbon burning. Has anybody done work on that?

\section{Discussion}

M. Cantiello: Yes, thermohaline mixing should occur during off-center carbon burning. I think this problem has been studied by Thomas (1965).

P. Woitke: To what extent is thermohaline mixing included in current standard stellar evolution codes (Iben, Lattanzio...)? Are you the first one who has solved this consistently?

M. Cantiello: I think it is not standard in most of stellar evolution codes at the moment, but thermohaline mixing has been implemented in our code in 2001 (Wellstein 
et al.) following the prescription of Kippenhahn (1980). Charbonnel \& Zahn (2007) studied the problem of thermohaline mixing driving the RGB consistently.

C. Charbonnel: Comment: Observations of ${ }^{12} C /{ }^{13} C$ give very strong constraints on the efficiency of thermohaline convection in RGB stars. Question: Thermohaline convection should have destroyed most of the ${ }^{3} \mathrm{He}$ in a $1 \mathrm{M}_{\odot}$ star on the RGB. So how can you have thermohaline convection on the early-AGB for such a star, if the ${ }^{3} H e$ reservoir is empty?

M. Cantiello: Yes. Thermohaline mixing can be present during core He burning and on the AGB and TP-AGB if ${ }^{3} \mathrm{He}$ reservoir is not empty. This is the case in our models since we use Kippenhahn's prescription. Also stars that avoid thermohaline mixing on RGB ( $\sim 5 \%$ of low mass stars) could show thermohaline mixing in subsequent phases. Interestingly there are observations of a few AGB stars which show surface abundances in agreement with thermohaline mixing occurring during this phase. 